\title{
The "prawn-in-the-tube" procedure in the cuttlefish: Habituation or passive avoidance learning?
}

\author{
Véronique Agin, ${ }^{1}$ Raymond Chichery, Ludovic Dickel, and Marie-Paule Chichery \\ Laboratoire de Psychophysiologie, Université de Caen, 14032 Caen Cedex, France; C.R.E.C., 14530 Luc-sur-Mer, France
}

\begin{abstract}
This study examines whether or not habituation contributes to the regulation of the inhibition of predatory behavior observed during the "prawn-in-the-tube" training procedure. When presented with prawns that are visible behind glass but untouchable, cuttlefish promptly learn to inhibit their capture attempts. The first three experiments demonstrated that the acquired response in the course of training cannot be dishabituated. The fourth experiment demonstrated that the repeated application of a brief visual prawn stimulus, one that is terminated before the cuttlefish can strike, decreases attack latencies. Taken together, the results of this study establish that habituation does not play a significant role in this learning task. In fact, the present findings strengthen the results of previous studies suggesting that passive avoidance learning contributes to the regulation of the inhibition of predatory behavior.
\end{abstract}

In recent years, considerable progress has been made in identifying some of the elementary cellular and molecular mechanisms involved in learning and memory in various gastropod molluscs (Brown 1997; Benjamin et al. 2000; Zhang et al. 2003; Brembs et al. 2004; Crow 2004). However, there is a need for other models, with a more complex central nervous system (CNS), suited to cellular, molecular, and network analysis. For a comparative analysis of this kind, cephalopod molluscs (cuttlefish, squid, octopuses) are ideal models: They have developed the most sophisticated CNS of all the invertebrates and exhibit unexpected behavioral abilities that are sometimes equal to those of lower vertebrates (Packard 1972; Fiorito and Scotto 1992; Boal 1996; Boal et al. 2000).

Cuttlefish actively prey upon shrimp and capture them by shooting out their two tentacles for a strike. This behavior is visually driven in the cuttlefish (Messenger 1968). It has been extensively studied in laboratory conditions: A shrimp is placed in a glass tube in the middle of an experimental tank. The cuttlefish then attacks its prey but does not obtain it (Messenger 1971, 1973; Chichery and Chichery 1992; Dickel et al. 2000). Under these conditions, Sepia officinalis promptly learns to inhibit the predatory motor pattern, i.e., the number of capture attempts (tentacle strikes) decreases with stimulus presentations. Messenger (1973) clearly demonstrated that this waning was not the result of motor fatigue or of a temporary incapacity to make a tentacle strike, and Agin et al. (1998) showed that it was not due to nonspecific effects such as stress or "contextual fear" induced by the manipulation of the experimental apparatus.

Nonassociative learning (habituation), or associative learning (passive avoidance learning), could contribute to the regulation of the waning of capture attempts. Habituation is classically defined as the relatively persistent waning of a response as a result of repeated stimulation that is not followed by any kind of reinforcement (Thorpe 1963). Conversely, the decreased response to repeated stimulation observed in passive avoidance learning is due to the association of this behavior with consecutive, aversive, painful stimuli. Messenger (1973) demonstrated that the level of striking was significantly lowered when negative reinforcement was enhanced, and was significantly raised when

\footnotetext{
1Corresponding author.

E-mail veronique.agin@unicaen.fr; fax 33-1-31565600.

Article published online ahead of print. Article and publication date are at http://www.learnmem.org/cgi/doi/10.1101/lm.90106.
}

negative reinforcement was reduced or when positive reinforcement was given. Because of this, Messenger (1973) suggested that the waning of the response of cuttlefish to prawns in a glass tube cannot be habituation but is, rather, an example of what Thorpe (1963) terms associative learning. There are numerous and various sensory receptors in the suckers of the tentacles (Graziadei 1964). Therefore, the tentacles striking the tube may deliver a "pain" message to the brain. This pain would then be associated with the prawns in the tube. However, it could be argued that we are dealing with a mixture of associative learning and habituation. In fact, cuttlefish whose tentacles had been removed exhibited a slight waning of attacks. This could be considered as the result of pure habituation, but we cannot exclude the possibility of a pain input from the tentacle stumps at every pseudo-strike. Because the "prawn-in-the-tube" procedure is now being used to explore the cellular and molecular correlates of behavioral plasticity (Agin et al. 2001, 2003; Bellanger et al. 2003), the precise nature of the task used in cuttlefish should be made clear. One critical test to distinguish between passive avoidance learning and habituation, which has not previously been done, concerns a fundamental empirical characteristic of behavior undergoing habituation, i.e., dishabituation, the ability of a strong, extra, or different stimulus to reverse the habituated response (Groves and Thompson 1970). In this study, the first three experiments examine whether the acquired response in the course of training could be dishabituated. For this, we used two different training procedures and two different dishabituatory stimuli. The fourth experiment examines whether or not the repeated application of a brief visual prawn stimulus, one that is terminated before the cuttlefish can strike, extends attack latency.

\section{Results}

\section{Experiment 1 (massed training)}

A schematic view of the experimental procedure is given in Figure 1. Figure 2 shows that the level of striking declined with time. Indeed, the Friedman test revealed that the number of strikes declined with each successive 2 -min block $(\mathrm{Fr}=27.425$, $P<0.001)$. The Wilcoxon signed-ranks tests confirmed that the number of tentacle strikes declined significantly between the first and fifth 2-min block $(\mathrm{Z}=-2.539, P<0.05)$. These results show a good acquisition of the task at the end of the first five 2-min blocks. By the end of the 10th minute of presentation of the glass 

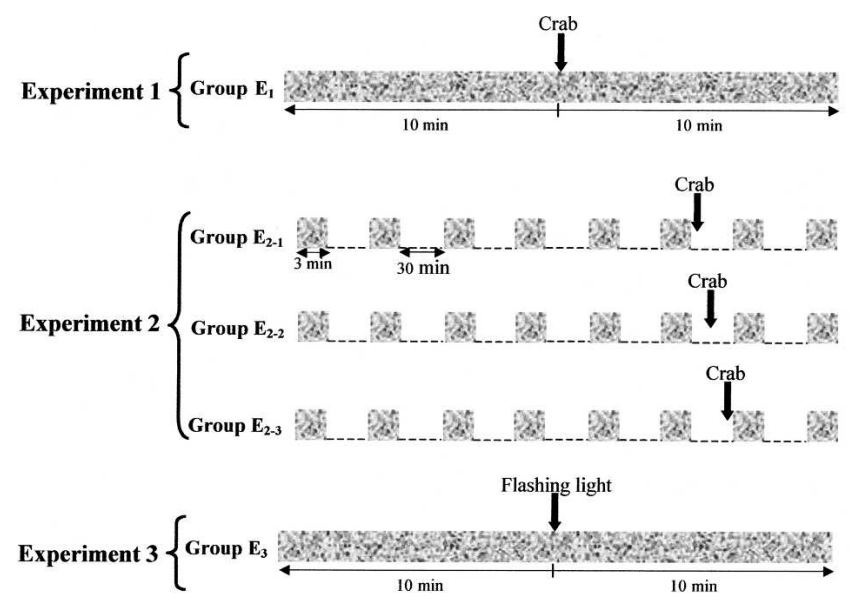

Experiment $4\left\{\right.$ Group E $_{4}$

Figure 1. Schematic representation of the experimental procedure. (Experiments 1 and 3) Massed training. The glass tube containing the prey was opened to the view of the cuttlefish for a single presentation of 20 min (gray rectangles). At the end of the 10th minute of training, experimental cuttlefish (groups $\mathrm{E}_{1}$ and $\mathrm{E}_{3}$ ) were given the arousing stimulation (crab: Experiment 1, flashing light: Experiment 3; see arrows). (Experiment 2) Spaced training. Cuttlefish were given eight presentations of the glass tube containing the prey (gray squares), lasting 3 min each, spaced at 30-min intervals (dotted lines). Twenty-nine minutes $30 \mathrm{sec}$ (group $E_{2-1}$ ), 15 min (group $E_{2-2}$ ), or $30 \mathrm{sec}\left(\right.$ group $E_{2-3}$ ) before the start of the seventh presentation, experimental cuttlefish were given the arousing stimulation (crab, see arrows). (Experiment 4) Fifty-six applications (black squares) of a brief visual prawn stimulus (shrimp). Shrimp were presented seven times (each $30 \mathrm{sec}$, see arrows) for a period of $3 \mathrm{~min}$, each of eight periods being separated by a rest interval of $30 \mathrm{~min}$ (dotted lines).

tube, the cuttlefish had significantly acquired the task, and so they were given the arousing stimulus (crab). When shown the crab, they promptly exhibited typical characteristics of predatory behavior: prey detection, orientation, chase, frontal positioning with respect to the prey accompanied by ocular convergence, and attempts at prey capture (the crab was removed just before they could seize it). Thirty seconds after the presentation of the crab, the training phase began again with the glass tube. There was no increase in response after the introduction of the crab (2-min blocks T6-T10) compared with the response during 2-min block T5 (Friedman test, $\mathrm{Fr}=2, P=0.849$ ). This means that the additional event did not produce dishabituation of the attenuated response.

\section{Experiment 2 (spaced training)}

A schematic view of the experimental procedure is given in Figure 1. As can be seen in Figure 3, the level of striking declined in the course of training. Indeed, the Friedman test revealed a significant decrease in tentacle strikes at each successive presentation of the glass tube (group $\mathrm{E}_{2-1}: \mathrm{Fr}=43.231, P<0.001$; group $\mathrm{E}_{2-2}: \mathrm{Fr}=24.302, P<0.001$; group $\mathrm{E}_{2-3}: \mathrm{Fr}=23.469, P<0.001$ ). Post hoc tests confirmed that progressive acquisition of the task occurred over the course of the first six presentations of the glass tube (group $\mathrm{E}_{2-1}: \mathrm{Z}=-3.183, P<0.01$; group $\mathrm{E}_{2-2}$ : $\mathrm{Z}=-2.666$, $P<0.01$; group $\left.\mathrm{E}_{2-3}: \mathrm{Z}=-2.371, P<0.05\right)$. Since the task had been significantly acquired by the end of the sixth presentation of the glass tube, the arousing stimulus (crab) was introduced into the experimental cuttlefish's tank $29 \mathrm{~min} 30 \mathrm{sec}$ before the start of the seventh presentation of the glass tube for group $\mathrm{E}_{2-1}$, 15 min before for group $E_{2-2}$, and 30 sec before for group $E_{2-3}$. As previously described, cuttlefish that were shown the crab tried to attack it within a few seconds. After the presentation of the crab, cuttlefish from the three experimental groups showed no significant increase in response to the prey enclosed in the tube compared with their response in the previous phase of training: Comparison of the number of tentacle strikes of the sixth, seventh, and eighth training sessions revealed that the presentation of the crab did not produce dishabituation of the inhibition of predatory behavior (Friedman tests, group $\mathrm{E}_{2-1}: \mathrm{Fr}=1.077, P=0.584$; group $\mathrm{E}_{2-2}: \mathrm{Fr}=2.722, P=0.256$; group $\mathrm{E}_{2-3}: \mathrm{Fr}=0.929$, $P=0.629)$.

\section{Experiment 3 (massed training)}

A schematic view of the experimental procedure is given in Figure 1. Figure 4 shows that there was a decrease in the number of strikes in the course of the first $10 \mathrm{~min}$ (Friedman test; $\mathrm{Fr}=23.657, P<0.001)$. Significant acquisition of the task was indeed present at the fifth 2-min block (Wilcoxon signed-ranks test; $\mathrm{Z}=-2.375, P<0.05)$. As in Experiment 1 , experimental cuttlefish were given the arousing stimulus (flashing light) after the end of the 10th minute of the training phase. In this case, all the cuttlefish showed changes of color over the entire body, with pronounced opening of the pupil, which demonstrated that the flashing light was an effective stimulus. Nevertheless, the number of attempted attacks on inaccessible prawns during 2-min blocks T6-T10 was not greater than in 2-min block T5 (Friedman test, $\mathrm{Fr}=9.531, P=0.09$ ). Therefore, despite the use of an arousing stimulus of another modality, we were unable to produce dishabituation of the inhibition of predatory behavior; for this reason, we did not try the spaced procedure with the flashing light.

\section{Experiment 4}

A schematic view of the experimental procedure is given in Figure 1 . When shown the shrimp $(56 \times$ over eight periods of $3 \mathrm{~min}$ each), all the cuttlefish tried to capture it within a few seconds (Fig. 5); the shrimp was removed just before they could seize it. Surprisingly, attack latencies gradually decreased from the beginning to the end of the experiment $(\mathrm{Fr}=16.592, P<0.05)$. The Wilcoxon signed-ranks tests confirmed that the attack latencies declined significantly between the second and eighth period $(\mathrm{Z}=-2.191, P<0.05)$. This experiment showed that removing the prey from the tank immediately after an initial attack did not prevent the cuttlefish from attacking the prey in the 55 subsequent presentations.

\section{Discussion}

We were unable to obtain dishabituation of the inhibition of predatory behavior (Experiments 1-3), despite the use of two

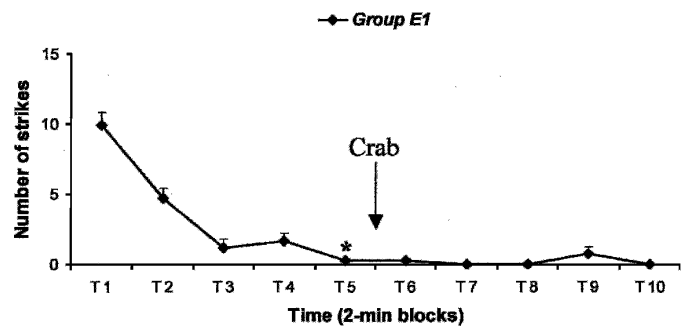

Figure 2. Massed training. The glass tube containing the prey was opened to the view of the cuttlefish for a single presentation of 20 min (ten 2-min blocks). At the end of the 10th minute of training, experimental cuttlefish (group $\mathrm{E}_{1}$ ) were given the arousing stimulation (crab). Arrow indicates the interval in which the arousing event occurred in the experimental group. Error bars indicate SEM. Asterisk indicates significant acquisition of the task (Wilcoxon signed-ranks test, $P<0.05, \mathrm{n}=8$ ). 


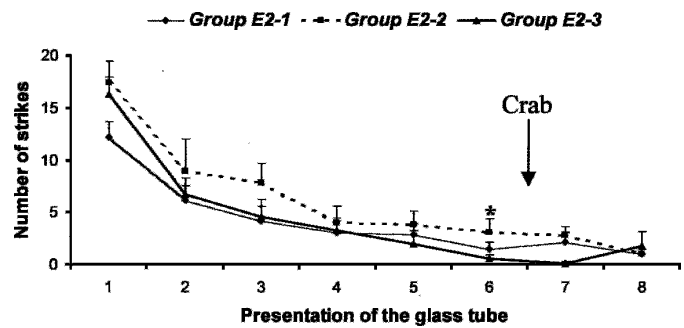

Figure 3. Spaced training. Cuttlefish were given eight presentations of the glass tube containing the prey, lasting 3 min each, spaced at 30-min intervals. Twenty-nine minutes $30 \mathrm{sec}$ (group $\left.\mathrm{E}_{2-1}\right), 15 \mathrm{~min}$ (group $\mathrm{E}_{2-2}$ ), or $30 \mathrm{sec}$ (group $\mathrm{E}_{2-3}$ ) before the start of the seventh presentation, cuttlefish were given the arousing stimulation (crab). Arrow indicates the interval in which the arousing event occurred in the experimental group. Error bars indicate SEM. Asterisk indicates significant acquisition of the task (Wilcoxon signed-ranks test, group $\mathrm{E}_{2-1}: P<0.01, \mathrm{n}=13$; group $\mathrm{E}_{2-2}: P<0.01, \mathrm{n}=9$; group $\mathrm{E}_{2-3}: P<0.05, \mathrm{n}=7$ ).

different training procedures and two different dishabituatory stimuli. The inability to demonstrate dishabituation can be explained by various factors. First, the choice of candidate dishabituation stimulus is an awkward parameter. Studies on dishabituation showed that one stimulus can be considered as an effective dishabituator when it induces a response, different, or not, from the response produced by the habituatory stimulus. In other words, all stimuli, that are sufficiently intense to produce an increase in the state of vigilance, are able to produce dishabituation of the habituated response (Groves and Thompson 1970; Mongeluzi and Frost 2000; Aoyama and McSweeney 2001). So, the absence of dishabituation in our experiments cannot be due to chosen dishabituatory stimuli (crab, flashing light) because an increase in the state of arousal of the cuttlefish was universally observed with the two stimuli. Moreover, experimental cuttlefish systematically seized and ingested accessible shrimps that were offered at the end of the tests. Consequently, the inhibition of predatory behavior after the arousing events cannot be attributed to surprise or fear of these stimuli. Second, the difficulty in demonstrating dishabituation might be due to the point at which the arousing stimulus is presented in the course of training and subsequent testing (re-exposure to trained stimulus). In studies with rats, Groves and Thompson (1970) trained animals to inhibit startle response to repeated acoustic stimulation, presenting 17 habituation stimuli at 1-min intervals; they reported that the rats showed recovery of the response immediately after a novel stimulus (flashing light) given between the 14th and the 15th habituation trial. Rats given a 30-min session in a running wheel each day over a 20-d training period (Aoyama and McSweeney 2001) showed dishabituation immediately after a brief extra event (the application of a brake or a flashing of the house light) lasting $5 \mathrm{sec}$, given $20 \mathrm{~min} 55 \mathrm{sec}$ after the beginning of a free running session. In Aplysia, Marcus et al. (1988) obtained an increase in siphon withdrawal reflex $90 \mathrm{sec}$ after the administration of an arousing stimulus (mild tactile stimulus) given just after habituation (20 habituation stimuli at 30-sec intervals). In Tritonia, dishabituation of the number of swim cycles (seven habituation sessions, at 4-h intervals, in which a salt stimulus was delivered to the tail every $2 \mathrm{~min}$ ) was demonstrated with a single dishabituatory salt stimulus to the head applied $5 \mathrm{~min}$ before the seventh session (Mongeluzi and Frost 2000). However, in the same organism, massed procedure failed to produce dishabituation (12 tail stimuli, 10-min intervals, with a dishabituatory salt stimulus to the anterior body 2 min before the 11th session (Frost et al. 1996). In a cephalopod, the squid Lolliguncula brevis, Long et al. (1989) trained animals to inhibit the number of escape jets and the production of a chro- matophore ring pattern in response to brief (5-sec) presentations to models of predators. Dishabituation was examined after habituating the squids to a model shark for 15 trials (1-min intervals) and then presenting a threat stimulus (waving a hand near the squid) followed by 15 trials of the same shark presentations. Dishabituation was partially demonstrated. In fact, after presentation of the threat stimulus, the squids showed elevated ring pattern responses (ring) to the next several presentations of the model shark, but the effects for escape jets were not statistically significant.

These studies give us no indication of an optimal time for the presentation of a novel stimulus and re-exposure to trained stimulus in the cuttlefish. For this reason, in our experiments, the time at which arousing stimuli were presented was varied according to conditioning. It is unlikely, therefore, that presentation of the arousing stimulus and testing were not carried out at the right time. Finally, the inability to produce dishabituation in our experiments might be explained by interference of simultaneous sensitization induced during training. In fact, Mongeluzi and Frost (2000) reported that dishabituation was difficult to demonstrate in Tritonia because of the presence of short-term sensitization induced during habituation training (Willows and Dorset 1975; Frost et al. 1996). The simplest explanation for the occlusion of dishabituation by sensitization is that the two processes share common underlying mechanisms (Groves and Thompson 1970). Could sensitization mask dishabituation of the inhibition of predatory behavior in cuttlefish? In our opinion, it is highly improbable: Our spaced training protocol, with a rest period between each training session, allowed us to test dishabituation when the inhibition of predatory behavior has been acquired but after recovery from a hypothetical short-term sensitization. Moreover, several studies have concluded that the processes underlying dishabituation and sensitization are completely separate (Rankin and Carew 1988; Erlich et al. 1992; Wright et al. 1992; Sahley et al. 1994). In Experiment 4, the repeated application of a brief visual prawn stimulus, one that was terminated before the cuttlefish could strike, did not extend attack latencies, as expected if habituation were occurring. Instead, these stimuli decreased attack latencies. This last control, combined with the other experiments, significantly strengthens the idea that habituation is not a part of the decrease in responsiveness observed during the prawn-in-the-tube procedure.

In conclusion, the findings of this study, with those of Messenger (1973), strongly argue for associative learning and, more precisely, for instrumental conditioning as a process of this learning: Acquired behavior is the result of pairing capture attempts with aversive consequences (tentacle strikes on the glass tube) (Messenger 1973). And in fact, the data show a definitive corre-

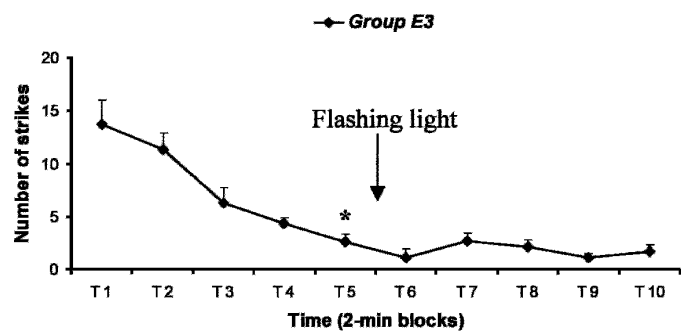

Figure 4. Massed training. The glass tube containing the prey was opened to the view of the cuttlefish for a single presentation of 20 min (ten 2-min blocks). At the end of the 10th minute of training, cuttlefish (group $\mathrm{E}_{3}$ ) were given the arousing stimulation (flashing light). Arrow indicates the interval in which the arousing event occurred in the experimental group. Error bars indicate SEM. Asterisk indicates significant acquisition of the task (Wilcoxon signed-ranks test, group $\mathrm{E}_{3}, P<0.05$, $n=7$ ). 


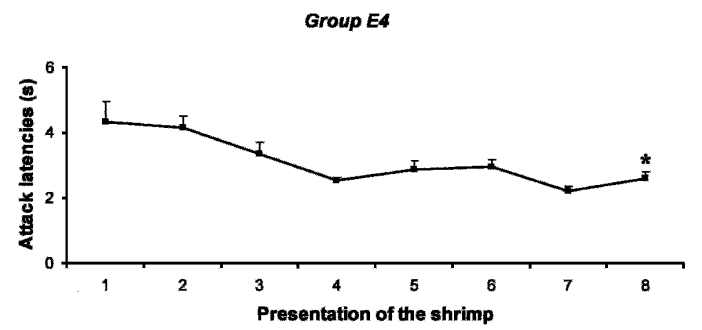

Figure 5. Fifty-six applications of a brief visual prawn stimulus (shrimp), one that is terminated before the cuttlefish (group $\mathrm{E}_{4}$ ) can strike. Shrimp were presented seven times (each $30 \mathrm{sec}$ ) for a period of $3 \mathrm{~min}$, each of eight periods being separated by a rest interval of $30 \mathrm{~min}$. Error bars indicate SEM. Asterisk indicates significant decrease of the attack latency between the second and eighth period (Wilcoxon signed-ranks test, group $\mathrm{E}_{4}, P<0.05, \mathrm{n}=10$ ).

spondence between the inhibition of tentacle strike and passive avoidance learning, where the cuttlefish learns to suppress its predatory behavior in order to avoid punishment. In addition, the fact that experimental cuttlefish readily seized and consumed accessible shrimp at the end of the experiment suggests that cuttlefish could tell the difference between accessible and inaccessible prey. Are the cuttlefish progressively able to discriminate the glass tube? Could this learning be contextual? Further work will be required to test these ideas.

\section{Materials and Methods}

\section{Animals}

Adult cuttlefish (218-262 mm dorsal mantle length) of both sexes were collected by trawler several miles off the coast of Ouistreham (Northern France). They were, thereafter, housed in individual tanks $(80 \times 60 \times 40 \mathrm{~cm})$ with circulating seawater maintained at a temperature of $15^{\circ} \mathrm{C} \pm 1{ }^{\circ} \mathrm{C}$. They were fed daily ad libitum with live shrimp (Crangon crangon) and crabs (Carcinus mænas). Cuttlefish showing external scars or not eating regularly were discarded. After an acclimatization period of $3 \mathrm{~d}$, cuttlefish were assigned at random to six different experimental groups. All the cuttlefish were naive at the outset, and each was used only once. Good ethical standards have been followed in the care, housing, and use of the animal subjects in our study.

\section{Procedure}

The object of the first three experiments was to discover whether the inhibition of predatory behavior shows dishabituation when using an arousing stimulus in the course of training.

\section{Apparatus}

The experimental apparatus was identical to that described by Messenger $(1971,1973)$. To allow presentation of the prey to the cuttlefish for the timed training sessions, a glass tube containing the prey (five shrimp) was concealed within an opaque plastic tube and placed in the tank $4 \mathrm{~h}$ before the start of the experiment. Training sessions could then be timed by the gentle removal and replacement of the plastic cylinder, a process $3-4$ sec.

\section{Training paradigms}

Two different training paradigms (Messenger 1973), massed and spaced, are usually used to study retention of the task in cuttlefish (Chichery and Chichery 1992; Dickel et al. 2000; Agin et al. 2001, 2003; Bellanger et al. 2003). It is well known in psychology that spaced training, interposing a rest interval between multiple training sessions, produces stronger and longer-lasting memory than does massed training involving the same number of training sessions (Hintzman 1974; Tully et al. 1994; Menzel 1999). We use these two different procedures (Fig. 1) to produce first acquisition of the inhibition of predatory behavior and to examine afterward dishabituation of the response. The spaced procedure was used to dismiss the effect of a possible short-term sensitiza- tion that could interfere with attempts to produce dishabituation (Frost et al. 1996; Mongeluzi and Frost 2000).

\section{Dishabituation stimuli}

A novel stimulus, of the same or of another modality than the habituation stimulus, involving an increase in the state of arousal can be considered as a candidate for dishabituation (Groves and Thompson 1970). In cephalopods, the only arousing stimulus previously used was "waving a hand near the squid" (Long et al. 1989). We rejected this stimulus on the grounds that it cannot be consistently repeated, and that there is a risk of unintentional cueing. We therefore experimented with two other stimuli: the presentation of a crab (which is specific to the cuttlefish, since it is among its natural prey) and a flashing light. Predatory behavior varies depending on the prey, although the first phases of the attack are the same for both crab and shrimp: visual prey detection, orientation, chase, frontal positioning with respect to the prey accompanied by ocular convergence, and attempt at prey capture. With shrimp, prey capable of a rapid escape, capture is achieved by projection of the two tentacles; in the case of less mobile prey, such as crab, capture is achieved by jumping on the prey (Messenger 1968). In our experiments (Experiments 1 and 2) (Fig. 1), however, the live crab was tied to a thread and, in each case, was removed just before it could be seized by the cuttlefish. The flashing light employed as a dishabituatory stimulus in Experiment 3 (Fig. 1) might be considered as an unspecific dishabituatory stimulus, since it has been widely employed on different models (Groves and Thompson 1970; Post and von der Emde 1999; Aoyama and McSweeney 2001), but we know that visual modality is much used by cuttlefish (Messenger 1968).

\section{Experimental design}

The final form of the experiment involved five dishabituation protocols, with different intertrial intervals for the training stimulus, different intervals between the training stimulus and the novel stimulus, different intervals between the novel stimulus and the test stimulus, and differences in modality of the arousing stimuli. In the first experiment (Fig. 1), we chose the massed procedure: The opaque plastic cylinder was removed for a single period of $20 \mathrm{~min}$, during which time the prey was visible to the cuttlefish and the number of strikes was counted. Earlier experiments have shown that the inhibition of predatory behavior is an acquired response and is stable at the end of the 10th minute of the training phase (Agin et al. 2003); the chronometer was therefore stopped at the end of the 10th minute, and the cuttlefish (group $\mathrm{E}_{1}, \mathrm{n}=8$ ) were given the arousing stimulus (crab). Thirty seconds after the presentation of the crab, the chronometer was started up again for the last $10 \mathrm{~min}$ of presentation period of the prey in the glass tube; the number of strikes was recorded to assess the evidence of dishabituation.

In the second experiment (Fig. 1), the spaced procedure was used. The glass tube was presented eight times, for a period of 3 min, each presentation being separated by a rest interval of 30 min. During each presentation of the tube, the number of capture attempts (tentacle strikes) was counted. In earlier studies, we showed that the inhibition of predatory behavior is an acquired response and stable at the end of the sixth presentation of the glass tube (Chichery and Chichery 1992; Agin et al. 2001). The experimental cuttlefish were therefore given the arousing stimulus (crab) between the sixth and the seventh presentation: the first group (group $\mathrm{E}_{2-1}, \mathrm{n}=13$ ) exactly $30 \mathrm{sec}$ after being exposed to the training stimulus (i.e., the sixth presentation of the glass tube), the second group (group $\mathrm{E}_{2-2}, \mathrm{n}=9$ ) 15 min before being re-exposed to the training stimulus (i.e., the seventh presentation of the glass tube), and the third group (group $E_{2-3}, n=7$ ) $30 \mathrm{sec}$ before re-exposure. In the third experiment, cuttlefish (group $\mathrm{E}_{3}$, $\mathrm{n}=7$ ) (Fig. 1) were tested with the same training procedure as in the first experiment, except that in this case, the arousing stimulus was a flashing light (10-msec duration, 300 lux, Vareclar M, Alvar Electronic). Because the proposition that habituation could be related to the visual stimulus presentation per se cannot be excluded, we tested, in a fourth and last experiment (group $\mathrm{E}_{4}$, 
$\mathrm{n}=10$ ) (Fig. 1) whether the repeated application of a brief visual prawn stimulus, one that is terminated before the cuttlefish can strike, extends attack latency. A live shrimp attached to a thread was presented to the cuttlefish and systematically withdrawn just after an initial attack signified by frontal positioning with ocular convergence. The shrimp was presented seven times at 30-sec intervals over a period of $3 \mathrm{~min}$, followed by a rest period of 30 min. This routine of presentation and rest periods was continued until eight presentation periods had been completed, making a total of 56 presentations of the live shrimps. Attack latencies were recorded throughout the experiment.

\section{Analysis of behavioral data}

\section{Experiments $1-3$}

To verify the acquisition of the task before the presentation of the arousing stimulus, the acquisition performances within groups were first evaluated. For Experiments 1 and 3, the number of tentacle strikes was plotted in 2-min blocks (T1: $\min 1+\min 2$; T2: $\min 3+\min 4$; etc.), and the number of tentacle strikes was compared within the first five 2-min blocks (T1-T5). For Experiment 2, the number of tentacle strikes for each 3-min presentation of the glass tube was plotted, and the number of tentacle strikes was compared within the first six presentations. Friedman tests were used for multiple comparisons (Siegel and Castellan 1988). In significant cases, Wilcoxon signed-ranks tests for matched samples were performed as post hoc tests (Siegel and Castellan 1988). To examine a possible dishabituation of the acquired inhibition of predatory behavior, we tested the amplitude of response to prawn in the glass tube after the presentation of the arousing stimulus. The number of tentacle strikes observed after the presentation of the crab (Experiments 1 and 2, blocks T6-T10 and seventh to eighth presentation of the glass tube, respectively) or the flashing light (Experiment 3, blocks T6-T10) was compared with the number observed just before its presentation (Experiments 1 and 2, block T5 and sixth presentation of the glass tube, respectively; Experiment 3, block T5). Friedman tests were used for multiple comparisons. In significant cases, Wilcoxon signed-ranks tests for matched samples were performed as post hoc tests.

\section{Experiment 4}

To verify whether the repeated application of a visual prawn stimulus extends attack latency, attack latencies were compared within the eight periods of $3 \mathrm{~min}$. Friedman tests were used for multiple comparisons. In significant cases, Wilcoxon signedranks tests for matched samples were performed as post hoc tests. Data analyses were conducted with the Systat software package (version 5.02).

\section{Acknowledgments}

This research was supported by the "Ministère de la Recherche et de la Technologie" and the "Société de Secours des Amis des Sciences." We are grateful to C. Harris and J. Harris for helping to correct the English translation.

\section{References}

Agin, V., Dickel, L., Chichery, R., and Chichery, M.P. 1998. Evidence for a specific short-term memory in the cuttlefish, Sepia. Behav. Process. 43: $329-334$.

Agin, V., Chichery, R., and Chichery, M.P. 2001. Effects of learning on cytochrome oxidase activity in cuttlefish brain. NeuroReport 12: $113-116$.

Agin, V., Chichery, R., Maubert, E., and Chichery, M.P. 2003. Time-dependent effects of cycloheximide on long-term memory in the cuttlefish. Pharmacol. Biochem. Behav. 75: 141-146.

Aoyama, K. and McSweeney, F.K. 2001. Habituation contributes to within-session changes in free wheel running. J. Exp. Anal. Behav. 76: 289-302.

Bellanger, C., Dauphin, F., Chichery, M.P., and Chichery, R. 2003. Changes in cholinergic enzyme activities in the cuttlefish brain during memory formation. Physiol. Behav. 79: 749-756.
Benjamin, P.R., Staras, K., and Kemenes, G. 2000. A systems approach to the cellular analysis of associative learning in the pond snail Lymnaea. Learn. Mem. 7: 124-131.

Boal, J.G. 1996. A review of simultaneous visual discrimination as a method of training octopuses. Biol. Rev. Camb. Philos. Soc. 71: $157-190$.

Boal, J.G., Dunham, A.W., Williams, K.T., and Hanlon, R.T. 2000. Experimental evidence for spatial learning on octopuses (Octopus bimaculoides). J. Comp. Psychol. 114: 246-252.

Brembs, B., Baxter, D.A., and Byrne, J.H. 2004. Extending in vitro conditioning in Aplysia to analyze operant and classical processes in the same preparation. Learn. Mem. 11: 412-420.

Brown, G.D. 1997. Isolated-brain parallels to simple types of learning and memory in Tritonia. Physiol. Behav. 62: 509-518.

Chichery, R. and Chichery, M.P. 1992. Learning performances and aging in cuttlefish (Sepia officinalis). Exp. Gerontol. 27: 233-239.

Crow, T. 2004. Pavlovian conditioning of Hermissenda: Current cellular, molecular, and circuit perspectives. Learn. Mem. 11: 229-238.

Dickel, L., Boal, J.G., and Budelmann, B.U. 2000. The effect of early experience on learning and memory in cuttlefish. Dev. Psychobiol. 36: 101-110.

Erlich, J.S., Boulis, N.M., Karrer, T., and Sahley, C.L. 1992. Differential effects of serotonin depletion on sensitization and dishabituation in the leech, Hirudo medicinalis. J. Neurobiol. 23: 270-279.

Fiorito, G. and Scotto, P. 1992. Observational learning in Octopus vulgaris. Science 256: 545-546.

Frost, W.N., Brown, G.D., and Getting, P.A. 1996. Parametric features of habituation of swim cycle number in the marine mollusc Tritonia diomedea. Neurobiol. Learn. Mem. 65: 125-134.

Graziadei, P. 1964. Receptors in the sucker of the cuttlefish. Nature 203: $384-386$.

Groves, P.M. and Thompson, R.F. 1970. Habituation: A dual-process theory. Psychol. Rev. 77: 419-450.

Hintzman, D.L. 1974. Theoretical implications of the spacing effect. In Theories in cognitive psychology (ed. R.L. Solso), pp. 77-99. The Loyala Symposium, Hillsdale.

Long, T.M., Hanlon, R.T., Ter Maat, A., and Pinsker, H.M. 1989. Non-associative learning in the squid Lolliguncula Brevis (Mollusca, Cephalopoda). Mar. Behav. Physiol. 16: 1-9.

Marcus, E.A., Nolen, T.G., Rankin, C.H., and Carew, T.J. 1988. Behavioral dissociation of dishabituation, sensitization, and inhibition in Aplysia. Science 241: 210-213.

Menzel, R. 1999. Memory dynamics in the honeybee. J. Comp. Physiol. A 185: $323-340$

Messenger, J.B. 1968. The visual attack of the cuttlefish, Sepia officinalis. Anim. Behav. 16: 342-357.

. 1971. Two stage recovery of a response in Sepia. Nature 232: 202-203.

. 1973. Learning in the cuttlefish, Sepia. Anim. Behav. 21: 801-826.

Mongeluzi, D.L. and Frost, W.N. 2000. Dishabituation of the Tritonia escape swim. Learn. Mem. 7: 43-47.

Packard, A. 1972. Cephalopods and fish: the limits of convergence. Biol. Rev. 47: 241-307.

Post, N. and von der Emde, G. 1999. The "novelty response" in an electric fish: Response properties and habituation. Physiol. Behav. 68: $115-128$.

Rankin, C.H. and Carew, T.J. 1988. Dishabituation and sensitization emerge as separate processes during development in Aplysia. J. Neurosci. 8: 197-211.

Sahley, C.L., Modney, B.K., Boulis, N.M., and Muller, K.J. 1994. The s cell: An interneuron essential for sensitization and full dishabituation of leech shortening. J. Neurosci. 14: 6715-6721.

Siegel, S. and Castellan, N.J. 1988. Nonparametric statistics for the behavioral sciences, 2nd ed. McGraw-Hill, New York.

Thorpe, W.H. 1963. Learning and instinct in animals. Methuen, London.

Tully, T., Preat, T., Boynton, S.C., and Del Vecchio, M. 1994. Genetic dissection of consolidated memory in Drosphila. Cell 79: 35-47.

Willows, A.O.D. and Dorset, D.A. 1975. Evolution of swimming behavior in Tritonia and its neurophysiological correlates. J. Comp. Physiol. 100: 117-133.

Wright, W.G., McCance, E.F., Lu, T., and Carew, T.J. 1992. Delayed-onset sensitization emerges after dishabituation in developing Aplysia. Behav. Neural Biol. 57: 170-174.

Zhang, H., Wainwright, M., Byrne, J.H., and Cleary, L.J. 2003 Quantitation of contacts among sensory, motor, and serotonergic neurons in the pedal ganglion of Aplysia. Learn. Mem. 10: 387-393.

Received December 7, 2004; accepted in revised form November 3, 2005. 


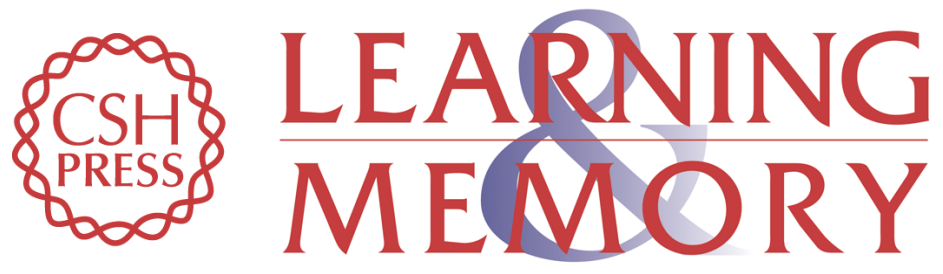

\section{The "prawn-in-the-tube" procedure in the cuttlefish: Habituation or passive avoidance learning?}

Véronique Agin, Raymond Chichery, Ludovic Dickel, et al.

Learn. Mem. 2006, 13:

Access the most recent version at doi:10.1101/lm.90106

References This article cites 32 articles, 9 of which can be accessed free at:

http://learnmem.cshlp.org/content/13/1/97.full.html\#ref-list-1

License

Email Alerting Receive free email alerts when new articles cite this article - sign up in the box at the Service top right corner of the article or click here. 